www.jmscr.igmpublication.org

Impact Factor 5.84

Index Copernicus Value: 83.27

ISSN (e)-2347-176x ISSN (p) 2455-0450

crossref DOI: _https://dx.doi.org/10.18535/jmscr/v5i5.58

Journal Of Medical Science And Clinical Research

IGM Publication

An Official Publication of IGM Publication

\title{
Original Research \\ Identification of Candidal Species from the Oral Cavity of Diabetic and Non-Diabetic Groups
}

\author{
Authors \\ Sonal Saigal $^{1}$, Ankur Bhargava ${ }^{2}$, Ahmad Danish Rehan ${ }^{3}$, Uddipan Kumar ${ }^{4}$, \\ Pragya Thakur ${ }^{5}$, Tasnim Kausar ${ }^{6}$
}

${ }^{1}$ Lecturer, Department of Oral Pathology, Microbiology \& Forensic Odontology, Dental Institute, Rajendra Institute Of Medical Sciences, Ranchi, Jharkhand.

${ }^{2}$ Reader, Department of Department of Oral Pathology, Microbiology \& Forensic Odontology, Hazaribagh

College Of Dental Sciences \& Hospital, Hazaribagh, Jharkhand

${ }^{3}$ Senior Lecturer, Department of Department of Oral Pathology, Microbiology \& Forensic Odontology,

Hazaribagh College Of Dental Sciences \& Hospital, Hazaribagh, Jharkhand

${ }^{4}$ Professor \& Head, Department of Department of Oral Pathology, Microbiology \& Forensic Odontology,

Hazaribagh College Of Dental Sciences \& Hospital, Hazaribagh, Jharkhand

${ }^{5}$ Post Graduate Student, Department of Conservative \& Endodontics, Hazaribagh College Of Dental

Sciences \& Hospital, Hazaribagh, Jharkhand

${ }^{6}$ Consultant Microbiologist, Clini Lab, Hazaribagh, Jharkhand

\section{Dr Sonal Saigal}

Address: C-3 Mahalashmi Apartment, SBI tower, Harihar Singh Road, Ranchi, Jharkhand, India

Email: drsonalsaigal@gmail.com, Contact No.:9723273639

\begin{abstract}
Diabetes mellitus is a common universal endocrine disorder with decreased host immunity towards infections. In these people the most common opportunistic infection is oral candidiasis. Oral candidiasis is most commonly caused by yeast like fungus Candida albicans. In healthy individuals these microorganisms are believed to be commensals but in diabetic patients, it forms severe colonization, even in the absence of any clinically evident oral candidiasis. This type of subclinical colonization can make them more prone to develop deeper mucosal colonization with further dissemination via blood. In the current study, we aimed to identify and compare the frequency of Candida spp. in the oral cavity of diabetic and non-diabetic groups.

Materials and Methods: Swabs were taken from the mouth of 60 participants and were cultured on Sabouraud dextrose agar (SDA) medium. The study was performed on two groups of diabetic patients $(n=30)$ and nondiabetics $(n=30)$ as the control group. The Candida spp. were identified by culture on CHROM agar Candida medium.

Results: The frequency of Candida spp .was higher in diabetic patients compared to non-diabetics. The most frequent Candida spp. in the diabetic patients were Candida albicans (\%36.66), C. krusei (\%16.66), C. glabrata (\%10.00), and C. tropcalis.(\%3.33). Likewise, C. albicans was the most frequent species (\%26.66) in the non-diabetic individuals. In this study, the results of both methods for identification of the isolates were consistent with each other.

Conclusion: Xerostomia and disturbance of physiological factors including $\mathrm{pH}$ and glucose can promote overgrowth of Candida flora in the oral cavity. These factors are considered important predisposing factors for oral candidiasis in diabetic patients.

Keywords: CHROM agar Candida, Diabetes mellitus, Oral candidiasis.
\end{abstract}




\section{Introduction}

Diabetes mellitus is a common and global epidemic in the new millennium, which is strongly related to lifestyle and economic change, caused chronic hyperglycemia with impairment of carbohydrate, lipid and protein metabolism resulting from defects in insulin secretion and action. The World Health Organization (WHO) has expected an increasing development of diabetes to more than 300 million by the year 2025; particularly, with type 2 diabetes mellitus

(1). However, T2DM was known as an adult-onset of diabetes in the past, but it has dramatically increasing more recently in young people and known for about $90 \%$ of the global incidence of diabetes and its complications ${ }^{(2)}$.

Oral candidiasis is a common opportunistic infection of the oral cavity caused by an overgrowth of candida species particularly Candida albicans ${ }^{[3]}$. Numerous risk factors such as age, gender, nutrition, oral hygiene, smoking, dentures, salivary $\mathrm{pH}$ disorder, and xerostomia (dry mouth) make diabetic patients more susceptible to oral candidiasis ${ }^{[4,5]}$. Diabetes mellitus (DM) is the most common endocrine metabolic disorder. Approximately $85-90 \%$ of diabetic patients are diagnosed with type 2 diabetes (resulting from insulin resistance); in these patients, salivary dysfunctions such as xerostomia, decreased salivary function, lichen planus, tooth decay, and periodontal diseases are common ${ }^{[6,7]}$. Among the reasons making diabetic patients more susceptible to oral candidiasis are high levels of salivary glucose, low secretion of saliva, impaired chemotaxis, and defect of phagocytosis due to polymorphonuclear leukocyte deficiency. The attachment of $\mathrm{C}$. albicans to the crystalline hydroxyapatite produces collagenolytic enzyme, which increases crystal solubility and consumes nitrogen of dentin collagen in DM patients ${ }^{[8,9,10]}$. Due to the upsurge in the level of non-albicans Candida species as well as azole-resistant isolates, finding a reliable diagnostic method is necessary for the treatment of Candida-related infections. The CHROM agar Candida is a chromogenic culture medium for the isolation and identification of Candida species based on colony color ${ }^{[11,12]}$. Herein, we aimed to determine the distribution of Candida species in the oral cavity with CHROM agar candida to evaluate and compared the amount of yeasts colonized in the oral cavity of diabetic with nondiabetic individuals. Our results can help with improving patient treatment outcomes.

\section{Materials and Methods}

A case control study was carried out in which samples were selected in a simple random sampling technique which included 30 cases of diabetics and 30 cases of age and gender matched controls (normal individuals). Informed consent from each person was taken and ethical clearance has been obtained for this study. A detailed history from the patient was taken which includes age, sex and duration of diabetes and the blood sugar level. The age groups of the samples were within $40-60$ years. A thorough intraoral examination was carried out for all patients. People were excluded from the study with the following factors: Patients with clinical lesions of oral candidiasis, patients wearing dentures, edentulous patients, patients with harmful oral habits, recent history of antibiotic therapy, acute and chronic diseases, endocrine disorders immunodeficiency diseases, nutritional deficiency diseases. The study groups consisted of DM patients with fasting serum glucose level of higher than $126 \mathrm{mg} / \mathrm{dl}$ and medical records of the glycosylated hemoglobin (HbA1c). Sample collection swabs were taken from the mouth of 60 subjects and were cultured on Sabouraud dextrose agar (SDA) medium. The plates were incubated for $72 \mathrm{~h}$ at $25^{\circ} \mathrm{C}$. Then, pure colonies were transferred on CHROM agar candida for the isolation and presumptive identification of Candida species. The Candida isolates were identified after incubation for $48 \mathrm{~h}$ at $37^{\circ} \mathrm{C}$. Light green colony color reveals $\mathrm{C}$. albicans, steel blue colonies C. tropicalis, large, fuzzy, and rosecolored colonies indicated C. krusei ${ }^{[11]}$. 


\section{Results}

From the samples of 30 diabetic patients and 30 controls, 20 (66.66\%) and $11(36.66 \%)$ were positive for Candida species, respectively. Table 1 exhibits identification of Candida species by growth on CHROM agar Candida medium. Of the 20 isolated Candida spp. in DM patients, 11 $(36.66 \%)$ were identified as Candida albicans, whereas C. dubliniensis, and C. parapsilosis were not isolated from any of the study groups. In the healthy subjects group, $08(26.66 \%)$ C. albicans were identified (Table 2). The current results indicated that colonization of Candida in the mouth of diabetic patients was more frequent in comparison with the non-diabetic group. In addition, C. albicans was the most prominent species isolated from the oral cavity of both groups.

Table 1. Identification of Candida species by growth on CHROMagar Candida medium

\begin{tabular}{|l|c|}
\hline Candida species & Colony color on CHROMagar \\
\hline C. albicans & Light green(19) \\
\hline C. tropicalis & Blue(1) \\
\hline C. krusei & Pink-purple(5) \\
\hline C. glabrata & Pink(1) \\
\hline C. kefyr & \\
\hline
\end{tabular}

Table 2. Frequency of Candida species colonization in the oral cavity of the diabetic patients and controls

\begin{tabular}{|l|c|c|}
\hline Candida spp. & Diabetic patients No.(\%) & Healthy subjects No.(\%) \\
\hline C. albicans & $11(36.66 \%)$ & $08(26.66 \%)$ \\
\hline C. krusei & $03(10 \%)$ & $02(6.66 \%)$ \\
\hline C. glabrata & $02(6.66 \%)$ & -- \\
\hline C. tropicalis & $01(3.33 \%)$ & -- \\
\hline C. kefyr & -- & $01(3.33 \%)$ \\
\hline C. krusei with C. Albicans & $02(6.66 \%)$ & -- \\
\hline C. glabrata with C. albicans & $01(3.33 \%)$ & -- \\
\hline Total & $20(66.66 \%)$ & $11(36.66 \%)$ \\
\hline
\end{tabular}

\section{Discussion}

Poor oral hygiene in diabetic patients may increase the level of Candida spp. as part of the oral flora and might affect the superficial and systemic fungal infections compared with healthy individuals ${ }^{[13-14]}$. In oral candidiasis, biofilm formation and overgrowth of Candida species are significantly higher in diabetic patients. A combination of host and fungal risk factors such as increased salivary glucose, decreased salivary $\mathrm{pH}$, salivary flow reduction, advancing age, dentures, smoking habits, irritation, and xerostomia facilitate Candida spp. colonization ${ }^{[15]}$.

Recently, resistance to antifungal agents has been reported in Candida species, especially in strains isolated from immunocompromised patients. Our objective was to compare the presence and colonization of Candida spp. in the saliva of diabetic patients and non-diabetic controls. We found higher incidence of Candida infection in diabetic patients. The increased candidal colonization in diabetic patients could be attributed to the promotion of the binding of Candida to epithelial cells and reduction of tissue resistance against infection. Likewise, salivary glucose and $\mathrm{pH}$ levels are correlated with the increased carriage rate of Candida in diabetic patients ${ }^{[16-18]}$. In the present study, diabetic patients aged 40-60 years old with high salivary glucose, low salivary $\mathrm{pH}$, history of xerostomia, and low oral hygiene showed more than 50 colonies of Candida in their mouth. Our data revealed that $20(66.66 \%)$ of diabetic patients were found to carry Candida spp. in their oral cavity compared with the non-diabetic controls $(26.66 \%)$, which was in accordance with the findings of the previous studies ${ }^{[19,20,21]}$. Although, among the Candida species, C. albicans has the 
highest frequency in the oral cavity, in the last two decades, the incidence of oral candidiasis with other species such as C. glabrata and C. krusei that are less sensitive to azole compounds has increased. C. dubliniensis is mostly detected in the oral cavity of patients infected with the human immunodeficiency virus (HIV) ${ }^{[22,23]}$. The differentiation between $\mathrm{C}$. dubliniensis and $\mathrm{C}$. albicans due to the high degree of phenotypic similarity remains a problem. This species produce a distinctive dark green color on CHROM agar Candida compared to C. albicans isolates, which are light green ${ }^{[24,25]}$. In evaluation of subjects with poorly controlled and wellcontrolled type 2 diabetes using CHROM agar Candida, Melton et al. reported that C. Dubliniensis was not found in oral samples, which were incubated at $42^{\circ} \mathrm{C}{ }^{[26]}$. In the current study, C. dubliniensis and C. parapsilosis were not detected in oral samples by CHROM agar candida.

\section{Conclusion}

The candidal load of oral mucusa in DM patients were found to be significantly higher than those of the control group. In the present study, the patients with high salivary glucose, low salivary $\mathrm{pH}$, history of xerostomia, and low oral hygiene showed more than 50 colonies of Candida in their mouth. Our data revealed that $66.66 \%$ of diabetic patients were found to carry Candida spp. in their oral cavity, and C. albicans was the most prominent species. Consideration of the possibility of oral Candida infections in DM patients is emphasized for improving patient treatment outcomes and reducing healthcare costs.

\section{References}

1. Diabetes--a global threat. Lancet. 2009;373(9677):1735.

2. WHO Technical Report Series. Prevention of diabetes mellitus; Geneva: WHO Publications; 1994.

3. Cannon RD, Holmes $\mathrm{AR}$, Mason $\mathrm{AB}$, Monk BC. Oral Candida: clearance, colonization, or candidiasis? J Dent Res. 1995; 74(5):1152-61.

4. Hopcraft MS, Tan C. Xerostomia: an update for clinicians. Aust Dent J. 2010; 55(3):238-44.

5. Guggenheimer J, Moore PA. Xerostomia: etiology, recognition and treatment. J Am Dent Assoc. 2003; 134(1):61-9.

6. Ship JA. Diabetes and oral health: an overview. J Am Dent Assoc.2003;134:410.

7. Vijan S. In the clinic: Type 2 diabetes. Ann Intern Med. 2010; 152(5):3-15.

8. Rotrosen D, Calderone RA, Edwards JE Jr. Adherence of Candida species to host tissues and plastic surfaces. Rev Infect Dis. 1986; 8(1):73-85.

9. Aly FZ, Blackwell CC, Mackenzie DA, Weir DM, Clarke BF. Factors influencing oral carriage of yeasts among individuals with diabetes mellitus. Epidemiol Infect. 1992; 109(03):507-18.

10. Samaranayake LP, MacFarlane TW. The adhesion of the yeast Candida albicans to epithelial cells of human origin in vitro. Arch Oral Biol. 1981; 26(10):815-20.

11. Jabra-Rizk MA, Brenner TM, Romagnoli M, Baqui AA, Merz WG, Falkler WA Jr, et al. Evaluation of a reformulated CHROMagar Candida. J Clin Microbiol. 2001; 39(5):2015-6.

12. Nadeem SG, Hakim ST, Kazmi SU. Use of CHROMagar Candida for the presumptive identification of Candida species directly from clinical specimens in resource-limited settings. Libyan J Med. 2010; 5(1):21-44.

13. Fisher BM, Lamey PJ, Samaranayake LP, MacFarlane TW, Frier BM. Carriage of Candida species in the oral cavity in diabetic patients: relationship to glycaemic control. J Oral Pathol. 1987; 16(5):282-4.

14. Guggenheimer J, Moore PA, Rossie K, Myers D, Mongelluzzo MB, Block HM, et al. Insulin-dependent diabetes mellitus and 
oral soft tissue pathologies. II. Prevalence and characteristics of Candida and candidal lesions. Oral Surg Oral Med Oral Pathol Oral Radiol Endodontol. 2000; 89(5):570-6.

15. Mohammadi F, Javaheri MR, Nekoeian S, Dehghan P. Identification of Candida species in the oral cavity of diabetic patients. Curr Med Mycol, 2016;2(2):1-7.

16. Samaranayake L, Hughes A, MacFarlane $\mathrm{T}$. The proteolytic potential of Candida albicans in human saliva supplemented with glucose. J Med Microbiol. 1984; 17(1):13-22.

17. Odds FC, Evans EG, Taylor MA, Wales JK. Prevalence of pathogenic yeasts and humoral antibodies to Candida in diabetic patients. J Clin Pathol. 1978; 31(9):840-4.

18. Knight L, Fletcher J. Growth of Candida albicans in saliva: stimulation by glucose associated with antibiotics, corticosteroids, and diabetes mellitus. J Infect Dis. 1971; 123(4):371-7.

19. Darwazeh AM, MacFarlane TW, McCuish A, Lamey PJ. Mixed salivary glucose levels and candidal carriage in patients with diabetes mellitus. J Oral Pathol Med. 1991; 20(6):280-3.

20. Willis AM, Coulter WA, Fulton CR, Hayes JR, Bell PM, Lamey PJ. Oral candidal carriage and infection in insulintreated diabetic patients. Diabet Med. 1999; 16(8):675-9.

21. Dorocka-Bobkowska B, Budtz-Jörgensen E, Włoch S. Non-insulin-dependent diabetes mellitus as a risk factor for denture stomatitis. J Oral Pathol Med. 1996; 25(8):411-5.

22. de Paula SB, Bartelli TF, Di Raimo V, Santos JP, Morey AT, Bosini MA, et al. Effect of eugenol on cell surface hydrophobicity, adhesion, and biofilm of Candida tropicalis and Candida dubliniensis isolated from oral cavity of
HIV-infected patients. Evid Based Complement Alternat Med. 2014; 2014:01-08.

23. Deorukhkar SC, Saini S, Mathew S. Nonalbicans Candida infection: an emerging threat. Interdiscip Perspect Infect Dis. 2014; 2014:1-7.

24. Gutierrez J, Morales P, Gonzalez MA, Quindos G. Candida dubliniensis, a new fungal pathogen. J Basic Microbiol. 2002; 42(3):207-27.

25. Deorukhkar SC, Saini S. Laboratory approach for diagnosis of candidiasis through ages. Int $\mathbf{J}$ Curr Microbiol Appl Sci. 2014; 3(1):206-18.

26. Melton JJ, Redding SW, Kirkpatrick WR, Reasner CA, Ocampo GL, Venkatesh A, et al. Recovery of Candida dubliniensis and other Candida species from the oral cavity of subjects with periodontitis who had well-controlled and poorly controlled type 2 diabetes: a pilot study. Spec Care Dentist. 2010; 30(6):230-4. 